Questions de communication

\title{
Du « public journalism » au « journalisme citoyen »
}

From public Journalisme to civic Journalism

Jean-François Tétu

\section{CpenEdition}

Journals

Édition électronique

URL : http://journals.openedition.org/questionsdecommunication/1681

DOI : 10.4000/questionsdecommunication.1681

ISSN : 2259-8901

\section{Éditeur}

Presses universitaires de Lorraine

\section{Édition imprimée}

Date de publication : 1 juillet 2008

Pagination : 71-88

ISBN : 978-2-86480-952-4

ISSN : 1633-5961

\section{Référence électronique}

Jean-François Tétu, « Du « public journalism » au « journalisme citoyen » », Questions de communication [En ligne], 13 | 2008, mis en ligne le 01 juillet 2010, consulté le 08 avril 2021. URL : http:// journals.openedition.org/questionsdecommunication/1681; DOI : https://doi.org/10.4000/ questionsdecommunication.1681 


\title{
$>$ DOSSIER
}

\author{
JEAN-FRANÇOIS TÉTU \\ Équipe de recherche de Lyon en sciences de l'information \\ et de la communication \\ Institut d'études politiques de Lyon \\ Université Lyon 2 \\ Jean-Francois.Tetu@univ-lyon2.fr
}

\section{DU «PUBLIC JOURNALISM » $A U \ll$ JOURNALISME CITOYEN »}

\begin{abstract}
Résumé. - La responsabilité sociale des médias et la responsabilité collective des journalistes apparaissent progressivement à la fin du $X X^{e}$ siècle, après plusieurs siècles de revendication de la liberté d'expression et d'impression. Elle est consécutive à l'affirmation du droit du public à l'information. Le public journalism, mouvement américain des années 90 , surtout ancré dans la presse locale, en est une illustration : il part de la crise de confiance du lectorat et des journalistes eux-mêmes, et propose une forme de journalisme fondé sur le consensus. Mais ce consensus risque de devenir un communautarisme qui éloigne le journalisme de la distance et de l'impartialité qui sont ses principes constitutifs. Aujourd'hui, la revendication « citoyenne » bascule des journalistes vers les lecteurs, d'où le développement des current event blogs.
\end{abstract}

Mots clés. - Presse, public journalism, civic journalism, liberté d'expression, responsabilité sociale, responsabilité collective, démocratie. 
a question de la responsabilité sociale des médias apparaît par étapes, dès la seconde moitié du $X X^{\circledR}$ siècle, dans une histoire dominée par un libéralisme sans cesse réaffirmé. C'est surtout à partir des années 80 qu'elle est revendiquée avec une grande netteté, après divers événements dont la couverture a fortement décrédibilisé la profession de journaliste et conduit à une large désaffection du public, notamment à l'égard de la presse écrite. La responsabilité, jusqu'alors considérée comme individuelle, apparaissait comme responsabilité collective, donnant naissance, par exemple en Amérique du Nord, à un mouvement nommé public journalism ou civic journalism, auquel est consacrée cette contribution. Plus récemment, le développement des blogs et de divers portails a induit une revendication « citoyenne » du contrôle de l'information. Parallèlement, en France, on assiste à la création de « chartes » dans les rédactions, à la prolifération du recours à la « déontologie », à l'« éthique », et à la mise en valeur du rôle des « médiateurs ». La taille de cet article interdit de s'interroger simultanément sur ces trois formes d'affirmation d'une responsabilité sociale, où il est souvent difficile de distinguer marketing ou affırmation d'une véritable responsabilité sociale et politique ou encore « citoyenne » du journalisme. Mais il est clair que l'identité du journalisme est directement mise en cause' par l'affirmation d'une responsabilité citoyenne (civic journalism), professionnelle (chartes) et éditoriale (médiateurs). Dans les trois cas, il s'agit d'une responsabilité collective : communauté «locale » dans le premier cas, corporation des journalistes et collectif d'une entreprise face au public dans le deuxième et le troisième. Tout cela, répétons-le, est récent et fort éloigné d'une histoire focalisée sur la liberté, qu'il nous faut d'abord rappeler rapidement.

\section{La liberté, source de la responsabilité dans l'espace public}

Les médias contemporains sont les héritiers d'un long combat pour la liberté de penser, puis la liberté d'expression, qui concerne d'abord le livre. Parmi toutes les traces de cette revendication, où s'ancre d'abord la lutte contre la censure religieuse, on peut relever l'exemple de l'Aeropagitica de John Milton en 1644 qui plaide « for the liberty of unlicensed printing $»^{2}$, au nom de la vérité chrétienne ( $«$ only the good can truly be free $»^{3}$ ). Cela

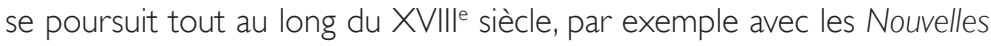
Ecclésiastiques, nées du rejet, par le peuple de France, de la condamnation des jansénistes dont témoigne la bulle Unigenitus.

\footnotetext{
Sur la question de l'identité du journalisme et de ses transformations en cours, voir www. surlejournalisme.com, et, notamment, les travaux de F. Le Cam et d'O.Trédan.

3 «Pour la liberté de la presse sans censure ».

3 « Seulement les bons peuvent être réellement libres ».
} 
La liberté «politique » ne s'affirme vraiment que sous le siècle des Lumières. Avant la Déclaration des droits de l'homme et du citoyen, deux textes constitutionnels : en Suède, en 1766, avec l'affirmation de la liberté de la presse, puis, en 1776, aux États-Unis, dans le Virginia's Bill of Rights (« the freedom of the press [...] can never be restrained but by despotic governments $»^{4}$ ), qui précède le Premier Amendement voté en 179|: « Le Congrès ne fera aucune loi restreignant la liberté de la parole ou de la presse $»$, ce qui rejoint les articles 10 et I I de la déclaration française. Cela se poursuit tout au long du XIXe siècle, notamment sous la plume de Karl Marx en 1842-43: «La presse libre [...] est le monde idéal qui jaillit perpétuellement du monde réel et, esprit toujours plus riche, y reflue pour le vivifier à nouveau » (in : Muhlmann, 2004 : 170).

Depuis la fameuse thèse de Jürgen Habermas (1962) sur l'espace public et les textes de Roger Chartier (1990) sur les origines culturelles de la Révolution française, nous sommes plus sensibles à la théorisation de cette liberté, notamment à partir des deux articles de Kant de 1784 et 1786. On peut d'ailleurs globalement voir dans les législations européennes du $\mathrm{XIX}$ siècle sur la presse la marque directe du caractère conservateur, despotique ou libéral des gouvernements successifs, dont témoigne la grande loi française de I88I sur la liberté de la presse. Mais l'attention portée à la démocratisation politique fait presque oublier la montée du capitalisme qui est une des causes directes de la crise de confiance dans la presse contemporaine, du double fait de la concentration et des conflits d'intérêts ${ }^{5}$, fortement dénoncés aux États-Unis dans diverses conférences ${ }^{6}$.

La doctrine libérale de l'information repose sur un principe hérité des Lumières: la raison universelle conduit à l'idée que nul ne peut avoir le monopole de la vérité, et donc à la contestation a priori du principe d'autorité7. Mais elle est prise dans une contradiction. D'une part, elle

4 «La liberté de la presse ne peut être restreinte que par des gouvernements despotiques ».

5 Sur la concentration, le premier texte américain est le rapport de la Commission Hutchins, en 1947, qui inspira ensuite les législations britannique et canadienne.

6 Notamment celle de R. M. Cohen, ancien producteur de CBS Evening News, et G. Roberts, directeur de la rédaction du New York Times (Barnouw et al., 1997). De nombreux chercheurs américains ont analysé les mécanismes de concentration et celui des cartels dans le monde des médias. Le plus souvent cité, et sans doute le précurseur, est B. H. Bagdikian (1983). Sur la question des conflits d'intérêts, et les manipulations, on peut évidemment se référer à N. Chomsky, mais aussi au très prolifique R.W. McChesney de I'Université d'Illinois ( 16 ouvrages sur ce sujet), et à E. Herman qui a cosigné différents travaux avec les deux précédents.

7 Ce qu'on voit bien en France avec les Ordonnances de 1944 et la loi Bichet de 1947 sur la distribution de la presse. 
présuppose qu'il n'y a pas de critère pour établir la vérité : on doit donc laisser parler quiconque peut aider à la vérité ${ }^{8}$, ce qui est à coup sûr une marque de défiance à l'égard de toute idée reçue; mais, d'autre part, et cela constitue une sorte de mythe contemporain, elle postule que, à l'instar de l'économie monétaire libérale, la bonne information finira par chasser la mauvaise. C'est là, comme on le verra, une des grandes ambiguïtés du public journalism parce que le public n'est ici qu'un autre nom du marché qui, sans qu'on le dise, devient le critère décisif de la vérités.

Cependant, le traumatisme de la Seconde Guerre mondiale et son cortège de propagande inversent la proposition libérale initiale : ce n'est pas tant le journaliste qui a le droit de dire ce qu'il pense, que le public qui a désormais droit à l'information. Ce virage a pris deux formes d'où émerge peu à peu la question de la responsabilité sociale de l'information : la presse remplit un service public ; elle a une responsabilité sociale ${ }^{10}$. On voit ici une évolution très sensible par rapport à la Déclaration universelle de l'ONu (article 19, 18 décembre 1948, www.un.org) centrée sur la liberté d'information, même si elle mentionnait l'exigence de la pluralité des sources et le souci moral des journalistes.

Au fond, la seconde moitié du $X X^{e}$ siècle voit un renversement sensible de perspective : la liberté n'est plus conçue comme un moyen de résister au despotisme, ce qui était au cœur de la pensée du XVIII| siècle, et l'État, tenu à distance par le Premier Amendement de la Constitution des ÉtatsUnis, devient le garant des libertés. On passe du droit de l'information au droit à l'information, formellement énoncé pendant le concile Vatican

8 On en trouve une autre illustration dans la campagne électorale de S. Royal en 2007 pour qui le débat public repose sur l'idée que chacun est un expert en ce qui le concerne.

9 Comment expliquer autrement qu'en 2007, un ancien coureur cycliste naguère convaincu de dopage, R. Virenque, par ailleurs très populaire auprès des supporters français du cyclisme, puisse devenir sans vergogne le « consultant » d'une grande station radiophonique.

${ }^{10}$ La première est américaine. On la trouve d'abord dans le rapport de la Commission Hutchins en 1947: «La presse doit savoir que ses erreurs et ses passions ont cessé d'appartenir au domaine privé pour devenir des dangers publics. Si elle se trompe, c'est l'opinion qu'elle trompe [...] la presse doit rester une activité libre et privée, donc humaine et faillible; et pourtant elle n'a plus le droit d'errer. Car elle remplit un service public ». Une mise en forme sensiblement postérieure est due, en 1963, à trois universitaires (Siebert et al., 1963), dont l'un au moins, W. Schramm, est par ailleurs, après E. Rogers et avec D. Lerner, un des promoteurs du « développementalisme » et un des inspirateurs de la politique de l'UNESCO pendant les années 60. Ces trois chercheurs proposent une typologie dont la légèreté historique et conceptuelle a de quoi faire frémir, mais dont le dernier type, la social responsability peut faire florès, car elle fournit une sorte de modèle de l'achèvement de l'évolution démocratique des médias en même temps qu'un retour aux origines de la pensée libérale. 
II dans l'Encyclique Pacem in terris qui mentionne explicitement (article 12) «le droit de tout être humain à une information objective ». Depuis lors, l'idée du droit à l'information s'est étendue, du fait de la croissance de l'audiovisuel, au droit à la communication, comme on le lit sous la plume de Jean d'Arcy, fondateur de l'Union européenne de radiodiffusion, en 1969. Cette évolution est à l'origine d'un mouvement très sensible à la fin du siècle parce qu'il constitue, en France, le fondement juridique de la libéralisation de l'audiovisuel (1982) et de la communication des collectivités locales dont on connaîtl'ampleurà partir des élections de 1982 et 1989, avant d'inspirer deux grands programmes gouvernementaux ${ }^{\prime \prime}$. Mais la question qui nous occupe ici est beaucoup plus limitée.

L'idée d'une responsabilité du journaliste est certes présente, notamment en France au moment où la profession tente de s'organiser, à partir de 1918 (Tétu, 2002), sous la forme des « droits » et « devoirs » du journaliste, mais on doit signaler que la revendication éthique est de type individuel et nullement politique et sociale : le journaliste, même lorsqu'il cesse de se penser comme écrivain, fait œuvre de création, intellectuelle, personnelle et individuelle. L'idée selon laquelle le journaliste doit être un « chien de garde » (watchdog) de la démocratie est beaucoup plus marquée aux États-Unis, où le journaliste se veut depuis longtemps un gardien des droits, et un pourfendeur des turpitudes. Mais la fin du XXe siècle conduit à une perte des anciens repères : la chute du mur de Berlin, la fin de I'URSS interdisent désormais toute pensée globale ; la guerre du Golfe montre une totale incurie des médias, incapables de toute investigation, et Saddam Hussein reste en place ; le retour aux identités, nationales et ethniques entraîne des guerres, des massacres et des violences qu'on croyait impossibles depuis la fin du génocide nazi. En France, le titre d'un éditorial d'Ignacio Ramonet dans Le Monde diplomatique du printemps 1992, «L'ère du soupçon », nomme le malaise : celui des journalistes perturbés par le scandale du sang contaminé, celui d'une opinion choquée par le traitement du faux charnier de Timisoara en 1989, puis par la couverture de la guerre du Golfe en 1991, pour ne prendre que ces deux exemples. Les médias français sortent de là discrédités ${ }^{12}$. Émergent alors le leitmotiv « éthique » dans les propos des journalistes, notamment dans les publications du syndicat national des journalistes, la multiplication soudaine des « chartes $»^{13}$ et le développement des médiateurs. Malgré cela, les journalistes français continuent de privilégier leur indépendance

1 'Le PAGSI (Plan d'action gouvernemental pour une société de l'information) lancé à l'Université d'Hourtin en 1998 par L. Jospin, puis le programme Reso 2007, de J.-P. Raffarin.

${ }_{12}^{12}$ Voir les sondages annuels de l'ifor pour La Croix et Télérama.

13 M. Prodhomme (2003) a montré qu'elles sont bien plus souvent à l'initiative des directions qu'à celle des journalistes, d'où un soupçon assez fort sur la dimension marketing de ces chartes, supposées renforcer l'image de marque, passablement écornée, de divers médias. 
(liberté de décision et niveau d'autonomie personnelle), alors que cette indépendance est très secondaire aux États-Unis où « la possibilité d'aider les autres » vient en première position depuis 197। (Abare McCane, $1992: 7)$.

\section{La naissance du public journalism : responsabilité nouvelle ou reconquête du lectorat}

La revendication d'un journalisme « public » ou « civique » est née d'un double constat aux États-Unis: d'une part, la baisse très sensible du nombre de journaux, notamment locaux (200 ont disparu en 20 ans), et du nombre global de lecteurs (20 millions de lecteurs en moins), et d'autre part, la forte chute de la participation aux élections américaines de 1988 et 1992 qui tombe aux environs de 40 \% (contre 65 \% en 1960), après des campagnes où l'information fut particulièrement déplorable, notamment dans l'affrontement Bush vs Dukakis, d'où une défiance forte du lectorat.

La situation américaine est analysée autrement par les pilotes de ce nouveau «mouvement » comme le nomme son fondateur Jay Rosen ${ }^{14}$ (1999), et rapidement diffusée et défendue par le Pew Center for Civic Journalism (PCC) $)^{15}$. II s'agit, selon ses promoteurs, d'une nouvelle approche du métier de journaliste qui, selon Thierry Watine (2003: 231), « vise à accroître l'utilité sociale des professionnels de l'information au sein de leur environnement immédiat afin de garantir un meilleur fonctionnement de la vie démocratique ». Les principaux initiateurs de ce mouvement sont, outre Jay Rosen, Arthur Charity (1995), et Davis Meritt (1995), rédacteur en chef du Wichita Eagle (Kansas). Ce mouvement a rapidement acquis une importance significative, du moins dans la presse américaine locale : une enquête du Pcc de juillet 200 I auprès des cadres de 360 quotidiens américains tirant à plus de 20000 exemplaires indique que $66 \%$ des éditeurs y adhèrent. Pourtant, à lire les textes qui en émanent, on ne peut qu'être surpris. Leurs auteurs semblent y découvrir que le journalisme est bien une construction sociale et symbolique de la réalitél ${ }^{6}$, prônent des méthodes, dont le sondage, dont on connaît depuis longtemps les biais ${ }^{17}$. Nouvelle forme de marketing médiatique? On peut enfin

\footnotetext{
${ }^{14}$ Professeur à l'université de New York, auteur de nombreuses publications. Son site comporte toutes les informations qui permettent de situer son parcours et ses ${ }_{15}$ publications : http://www.nyu.edu/gsas/dzept/journal/Faculty/bios/rosen/biography.htm.

${ }_{16}$ Voir informations sur le site : http://cpn.org/sections/affiliates/pew.html.

${ }^{16}$ Voir notamment E.Véron (1987). Ce type de réflexion est depuis longtemps attesté aux États-Unis par d'excellents travaux, notamment ceux de G.Tuchman (1978), H. Moloch et M. Lester (1974), ou encore M. Fishman (1988).

${ }^{17}$ Voir notamment P. Champagne (1990), après P. Bourdieu (1973).
} 
estimer que la participation directe à la résolution de problèmes sociaux généralement locaux éloigne ce journalisme de la distance constitutive du journalisme d'information. Peut-être, après tout, ce « mouvement » relève-til d'un changement global de paradigme comme le pensent Jean de Bonville et Jean Charron (Brin et al., 2004).

Reprenons d'abord le constat de Jay Rosen : la presse fait fausse route en matière d'information politique, notamment en période électorale ; elle est coupable de dérapages éthiques incessants (faux reportages, absence de contrôle des sources, ce dont témoignent quelques retentissants licenciements, rumeurs et rectificatifs repris par les blogueurs américains, ce qui entraîne la crise de confiance d'un public dont le cynisme et le désengagement politique vont croissant, puis la baisse inquiétante du lectorat, mais aussi le malaise, voire le découragement des journalistes eux-mêmes. II faut donc une « réforme », dit Jay Rosen, qui, sur certains points, va à l'encontre de la tradition américaine la plus forte : on sait assez, en effet, qu'à l'opposé du journalisme « de révérence » dont la France donne un piètre exemple, le journaliste américain, héritier des muckrackers ${ }^{18}$ et du stunt journalism ${ }^{19}$ du début du $X X^{e}$ siècle, est a priori critique à l'égard de la classe politique, fréquemment agressif à son égard quand il faut dénoncer ses comportements nocifs et démasquer ses turpitudes ; le journaliste américain « ne dîne pas en ville », ne passe pas ses vacances avec les notables du moment, etc. ${ }^{20}$. Les modèles du journalisme américain sont plutôt à chercher du côté de figures comme celle d'Edward R. Murrow dont les célèbres documentaires de 1953 et 1954 ont abattu Mac Carthy. Mais les choses ont changé : « Ces 15 ou 20 dernières années, dit James Fallows, beaucoup de journalistes sont soudain devenus riches grâce aux talk shows, aux émissions politiques à la télévision et aux tournées de conférences sur les campus. Or tous ces exercices réussissent particulièrement bien à ceux qui sont arrogants, provocateurs, cyniques $\rangle^{21}$. Or, dit Jay Rosen, il faut désormais remplacer la critique par la recherche des solutions. II faut évidemment remarquer qu'aucune des grandes figures du journalisme américain ne s'est jamais désintéressée des solutions, pas plus qu'en France un Albert Londres, mais ils ne considéraient pas que la solution fût de leur ressort, ni de leur rôle.

\footnotetext{
${ }^{18}$ Littéralement « fouille merde », expression utilisée par Th. Roosevelt dans un discours le 14 avril 1906. L'expression, très péjorative, fut ensuite reprise par les journalistes euxmêmes, dont Pulitzer et les journalistes de son New York World.

19 Littéralement « tour de force », utilisé aussi pour les publicités agressives (stunt publicity), a stunt man est un cascadeur. Le stunt journalism appartient à ce qu'on appelait plus généralement exposure journalism qui cherche à faire voir ce qu'on tient caché. Un bon exemple en est le reportage de N. Bly (1887), qui s'est fait enfermer à l'hôpital psychiatrique de Blackwell's Island pour en dénoncer l'état.

${ }^{20}$ Voir a contrario l'excellente analyse de R. Rieffel (1984).

${ }^{21}$ Cité par J.-S. Stehli, sur www.grainesdechangement, mai 2004.
} 


\section{Un journalisme de consensus}

C'est donc d'abord au nom de la démocratie qu'il faut changer les choses, d'où le nom, revendiqué, de journalisme « civique »: «Peutil exister un type de journalisme qui non seulement donne aux gens des nouvelles et des informations, mais les aide à faire leur travail de citoyen, un journalisme qui ne se contente pas d'accorder son attention à la question civique choquante du jour, mais qui incite les gens à passer à l'action, à s'engager, à penser que c'est à eux qu'il appartient de résoudre les problèmes, un journalisme qui considère les gens non pas comme des spectateurs, mais comme des participants ? II ne conseille pas à la presse de renoncer à son rôle de chien de garde mais y ajoute au contraire d'autres responsabilités ». L'essentiel de ces nouvelles responsabilités, et donc des objectifs à atteindre, est d'abord l'utilité de l'information, c'est-àdire que le journaliste « civique » doit d'abord aider le public à retrouver le sentiment qu'il peut faire quelque chose pour changer la situation et, au fond, stimuler la « démocratie », terme qui revient sans cesse dans les propos. II faut cesser d'être spectateur pour devenir acteur. Ce thème est explicitement repris, entre autres sujets, par Sandra Mims Rowe, exrédactrice en chef du Portland Oregonian: « Je préfère augmenter de $10 \%$ la participation électorale que gagner le prix Pulitzer ». Une autre formule, ou image, enfonce le clou : « On peut être guide sans renoncer à son rôle de chien de garde. Et ils [les journalistes] sont ravis d'abandonner leur rôle de loup ». Et cela est terriblement ambigu, aussi bien le terme de « guide » que l'idée de démocratie, qui semble alors signifier consensus, ou pire, domination par une majorité. Le consensus, en effet, revient lui sans cesse : « Le journalisme civique [...] s'efforce de couvrir le consensus [mis en italique par nous] aussi bien que les conflits, les succès comme les échecs, avec des articles qui pourront aider d'autres collectivités à faire face à des problèmes difficiles $\rangle^{22}$. Écarté, le journalisme au service de la démocratie par l'intégration des conflits, tel que l'a théorisé l'école de Chicago. Ce modèle se voue au nouveau culte du fait majoritaire. Le pire, peut-être, étant que les exemples le plus souvent proposés exaltent des

\footnotetext{
22 S. M. Rowe poursuit : « Le journalisme civique essaie de proposer de nouvelles formules de reportage mieux adaptées aux nouveaux modèles de gouvernement. Nombre de gouvernements locaux abandonnent le modèle "gagnant contre perdant" pour résoudre les problèmes locaux, au profit d'une approche basée davantage sur le consensus et dans laquelle tout le monde est gagnant. Comment peut-on équiper le journalisme pour y parvenir? Nous faisons du bon travail quand il s'agit de couvrir un conflit, de traquer les gens et de compter les points des gagnants et des perdants. Mais, si vous envoyez un reporter couvrir une réunion dont les participants sont tous d'accord sur un point, il y a de grandes chances pour qu'il rentre à son journal en annonçant à son patron qu'il ne s'est rien passé, qu'il n'y a pas matière à un article. Les reporters de la vie communautaire s'intéressent aux cas dans lesquels les responsables de la collectivité sont d'accord comme à ceux dans lesquels ils sont en désaccord. C'est quelque chose de nouveau ».
} 
causes « charitables » à la façon (pur miroir narcissique) dont, en France, l'animateur Pierre Bellemare faisait surgir l'émotion au son du «Vous êtes formidables ».

La pertinence de la démarche se fonde, bien entendu, surle succès évalué en termes quantitatifs : « En Californie, I'Orange County Register a expérimenté une nouvelle technique lors d'un reportage sur les enfants pauvres qui vivent dans des motels aménagés pour les indigents situés littéralement en face du parc de Disneyland [...]. La réaction de la collectivité a été extraordinaire. Cette expérience a permis de recueillir 200000 dollars de dons, 50 tonnes de produits alimentaires et 8000 jouets ». Or rien n'est dit sur les causes de la pauvreté locale incriminée : « Le journalisme civique vise à fournir aux gens des possibilités d'intervention afin de les amener à agir, et à encourager l'interactivité entre les journalistes et les citoyens. II cherche à créer un dialogue avec les lecteurs, au lieu de se borner à transmettre les informations en sens unique $[\ldots] »$. Thierry Watine donne pour premier exemple de ce journalisme l'opération de lutte contre la criminalité dans des quartiers très défavorisés « Taking back our neigbourhoods $\gg^{23}$ du journal Charlotte Observer (Charlotte, Caroline du Nord) : reportages, interviews, partenariat avec radio et TV locales, et recherche de solutions: «le taux de criminalité a chuté de $24 \%$ depuis 1994 et le nombre de crimes violents a diminué de $48 \% \gg »^{24}$.

Pour faciliter ce dialogue avec la population, les journaux nord-américains communiquèrent d'abord l'adresse électronique des journalistes à la fin des articles. La messagerie devint rapidement une nouvelle ressource, prompte à faire croire, comme les images du tsunami indonésien, au mythe singulier du « tous journalistes » : le Charlotte Observer s'est rendu compte que certaines de ses meilleures idées provenaient du courrier électronique adressé par ses lecteurs. Plus précisément encore, ce journalisme se met au service d'une politique qui ne fait l'objet d'aucun examen critique. En voici un exemple assez singulier : le PCcj a récemment fourni des fonds à la NHPR (New Hampshire Public Radio) pour la mise au point d'un programme permettant d'estimer les factures de gaz et d'électricité, afin de mieux faire comprendre au public le problème de la déréglementation des services de distribution.

23 «Pour récupérer nos quartiers ».

24 Séminaire « Le rôle des médias dans le développement local et régional », Vision 2025 , Saguenay Lac Saint Jean (Québec, 20 févr. 2004). 


\section{Du public comme audience au bien public}

« Faire comprendre au public ». L'expression explique pour une part l'autre nom de ce mouvement, le journalisme « public ». En effet, il y a une distinction à opérer entre le journalisme «civique » (= citoyen) et le journalisme «public » (au sens de « bien public »), ce que permettent de comprendre les textes déposés sur le très sérieux site mww.usinfo.state.gov/journals, où il est facile de comprendre que le vocable « public » n'a rien à voir avec Kant ou Habermas, mais désigne le journal au service de la collectivité. À propos d'un sondage effectué en 1992 par le News Journal de Wilmington (Delaware) sur la croissance économique de la localité25 : « Un rapport récapitulatif résume ainsi l'objectif que visait ce projet :"Cerner les grandes questions, solliciter le point de vue du public sur ces sujets et suivre l'évolution de ces dossiers" ». Autre exemple illustratif : «En 1993, le Register, quotidien de la ville de Des Moines (lowa), a donné pour consigne à tous ses journalistes d'interviewer en face à face au moins quatre "Américains moyens" pour découvrir ce qui les tracassait » (au total, il y eut 600 interviews). Un peu plus clair encore: «En 1993 et en 1994, le quotidien Star de la ville d'Indianapolis (Indiana) a fait faire un sondage de grande envergure sur les attitudes des habitants de la région en matière raciale et il a consacré une semaine à la présentation des résultats. La série d'articles de suivi reflétait "l'expérience vécue des citoyens, et non pas l'opinion d'un expert", ce qui était une grande nouveauté pour ce journal ». Le journal doit ainsi « soulager les inquiétudes du public », ce qui fonde l'argumentation de Davis Meritt: «[fournir] des informations pertinentes de nature à clarifier les valeurs fondamentales » et « s'exprimer clairement sur les convictions et les priorités qui sous-tendent chacun des problèmes qui se posent au public ». Et Paul Malamud de conclure : « À une époque obsédée par les images superficielles - les excentricités des vedettes et les scandales du jour - l'attention portée à l'homme de la rue et à ses petits soucis pourrait bien être un ballon d'oxygène pour la presse aussi bien que pour la société civile des États-Unis ». Certes le public américain reste plus friand des frasques de Paris Hilton que les journalistes qui en sont parfois excédés, mais faut-il remplacer les people par les avatars des Simpson? Le projet de Jay Rosen et Davis Meritt, faire des journaux les sages-femmes de la citoyenneté, semble conduire à de nouvelles formes de communautarisme, trouve un puissant relais auprès des autorités politiques américaines et sert de relance de la consommation médiatique.

Pour ne pas se leurrer sur des perspectives que les exemples précédents semblent caricaturer, il faut considérer que, selon le PCc], le journaliste a une obligation envers la vie publique qu'on peut résumer ainsi : la capacité

${ }^{25}$ Les citations suivantes sont extraites des textes de P. Malamud, http://usinfo.state.gov 
à choisir la meilleure chance de stimuler la prise de décision citoyenne et la compréhension des problèmes par le public ; la capacité à prendre l'initiative d'informer sur les principaux problèmes publics de telle façon que ce public ait de meilleures connaissances sur les solutions possibles et sur les valeurs qu'on peut engager dans des actions alternatives (Lambeth, 1998). Le public journalism doit donc être envisagé au sein des formes alternatives des médias qui se sont développées aux ÉtatsUnis et que Chris Atton (2002) résume en quatre positions: advocacy media, qui doivent sensibiliser les dirigeants publics ou privés aux sujets sociaux; grassroots media, qui cherchent à être au plus près des racines locales, au risque de populisme; accountability media, qui doivent rendre transparents les faits publics; lobbying media, qui visent les profits privés. La grande enquête de David Weaver et Cleveland Wilhoit (1986) sur les représentations de la fonction primordiale de l'information va dans le sens de l'accentuation de l'idée selon laquelle le journaliste doit rendre un service au public. Cette idée n'est pas une spécificité américaine et Rémy Rieffel (1984) a bien montré combien la fonction missionnaire est ancrée dans l'imaginaire des journalistes français, mais alors qu'elle repose - en France - sur une capacité intellectuelle propre du journaliste, elle ressemble, aux États-Unis, à une « écoute » du public. En témoigne le recours systématique du public journalism au sondage, alors que, en France, le sondage intervient lorsqu'on ne sait pas comment traiter un sujet. Dans l'Hexagone, la croissance du recours au sondage semble surtout indiquer le désarroi des journalistes dans l'interprétation de la vie publique.

\section{Action vs représentation : le rôle du journaliste}

Avant de dire un mot de l'héritage contemporain de ce mouvement, il convient de s'arrêter un instant sur trois caractéristiques insistantes : le refus de la séparation de la représentation et de l'action ; la volonté d'introduire un autre agenda et une autre hiérarchie de l'information; la volonté de servir le bien public défini comme bien commun du groupe local.

La première dimension (le primat de l'action) est ce qui est le plus vivement rejeté par ses détracteurs au nom de l'impartialité constitutive de ce que serait l'essence du journalisme. Bien loin de vouloir défendre ici une introuvable ontologie du journalisme, il faut rappeler d'un mot ce qui fonde la « distance » en journalisme. Le point de départ en est bien l'idée de l'opinion telle qu'elle s'est construite au XVIII' siècle. Dans le texte rappelé plus haut ( 1784,1786$)$, Kant établit fort clairement (double exemple de l'officier et du prêtre) la nécessité de fonder un espace des opinions, séparé de l'espace des actions. L'Aufklärung ne vise donc pas une action, mais un processus né de l'échange public de regards qui, eux, sont portés 
vers l'action ; les Lumières, c'est ce qui vient, non pas de l'action, mais de ce regard. Cela a été fortement repris par Hannah Arendt (1978 : 87), qui, commentant Kant, explique que « seul le spectateur, mais jamais l'acteur, sait vraiment de quoi il retourne »; seul le spectateur sait vraiment le sens de ce qui se passe, parce que « le fondement existentiel de sa perspective est son désintéressement, sa non-participation, son absence d'engagement ».

L'idée de l'impartialité en découle naturellement, parce qu'elle provient nécessairement d'un échange intersubjectif : « je nourris l'espoir », écrit Kant dans une lettre citée par Hannah Arendt (ibid. : 70) , « qu'en considérant ainsi, avec impartialité, mes jugements du point de vue des autres, je parviendrai à un troisième aperçu qui améliorera ma perspective antérieure », et Hannah Arendt (ibid.) de poursuivre : «On accède à l'impartialité en prenant en compte le point de vue des autres ; l'impartialité ne résulte pas d'une position supérieure qui $[\ldots]$ trancherait la querelle ». En somme, contrairement à l'élu qui reçoit mandat de ses électeurs pour agir dans la vie publique, le journaliste a mandat d'en construire une représentation, d'en chercher et d'en dire le sens, ce qui est une constante forte dans la vision classique du journalisme. Dans cette perspective, le tort du journaliste public ou civique est moins de perdre son indépendance lorsqu'il organise des forums ou propose des solutions, que de confondre son rôle avec celui de l'élu.

On sait que la plus forte critique portée à la définition kantienne de l'usage public de la raison vient de Marx, pour qui le regard politique est biaisé, aveugle à ce qui le détermine : les promesses du principe de publicité sont doublement fausses, d'abord parce que certains groupes sont exclus de fait de l'espace public, ensuite parce que les représentations sont trompeuses, entachées d'idéologie, ce que reprendra plus tard Louis Althusser (1970). C'est pour cela que Marx, qui défendait l'idée du public dans la Rheinische Zeitung d'octobre 1842 à mi-43, l'abandonne dans l'Idéologie allemande en 1845 au profit de l'idée de masse : «C'est en tant qu'elle n'est pas un public observateur et bavard que la masse est le moteur de l'histoire » (in : Muhlmann, $2004: 201)^{26}$.

\footnotetext{
${ }^{26}$ Reste que la distinction entre représentation et action demeure un point tout à fait incontournable dans l'histoire du journalisme: A. Londres porte «la plume» (et non le fer) « dans la plaie », et, avant lui, J.Vallès, journaliste, et insurgé, qui décrit ainsi L. Blanqui : « II laisse, d'une voix sereine, tomber des mots qui tranchent, et qui font sillon dans le cerveau des faubouriens, et sillon rouge dans la chair bourgeoise ». Cela n'est pas sans ambiguité, nous n'en voulons pour preuve le fait que, selon D. Reynié, le vote, en France, de la loi de I88I tient pour beaucoup au fait que les conservateurs y voyaient le moyen de transformer la violence réelle des foules en conflit symbolique d'opinions : « La police des formes cherche l'expulsion de l'opinion comme action » (Reynié, 1998 : 309). C'est, écrit ce dernier, la raison pour laquelle cette loi « autorise la libre impression d'un chant séditieux, mais elle interdit qu'il soit chanté dans la rue », ou encore interdit le cri parce que le cri « est situé dans la catégorie de l'action, non de l'opinion ».
} 
Prenons maintenant les deux autres dimensions du journalisme public, un «bien public » fondé sur une conscience commune des problèmes sociaux. Or, sur ce point, ce sont les théoriciens de l'école de Chicago qui fournissent les éléments de réflexion les plus précieux, à commencer par sa figure la plus remarquable, Robert E. Park. Dès sa thèse de 1904, ce dernier, s'inspirant de Gabriel Tarde (|90|), distingue la « conscience commune » (common consciousness, propre au « public ») de la « poussée commune » (common drive, propre à la foule), distinction que semblent bien méconnaître les chantres du journalisme public. En effet, la réflexion de Robert E. Park est largement inspirée de Georg Simmel (1908) dont il reprend deux idées fortes. La première est que le conflit est une forme de socialisation, qui intègre, au-delà des divisions qu'il révèle, ce que Robert E. Park reprend sous les deux formes d'« opposition » et de « compétition ». On est ici très loin du consensus du public journalism.

La seconde idée est encore plus intéressante, c'est la théorie, chez Georg Simmel, de l'« objectivation » : le conflit produit des « objets communs » qui pour tous ont le même sens, indépendamment des points de vue subjectifs; ces objets communs, qui sont construits par l'échange subjectif, ce sont les « faits ». Les conflits d'interprétation sont des conflits de « valeurs », et l'objectivation est ce qui permet de distinguer les faits et les valeurs. Sous cette perspective, l'erreur du public journalism est simplement de confondre les faits et les valeurs ${ }^{27}$. II faut cependant ajouter que, pour Robert E. Park (1938), au fil de sa réflexion, afin que le conflit puisse avoir cette fonction, il faut une intégration socioculturelle préalable, ce pourquoi il introduit plus tard le rôle majeur de la communication «comme principe d'intégration et de socialisation ». Et précisément, le journalisme américain, inventeur du human interest, produirait cette intégration. Encore faut-il distinguer, là dedans, ce qui est littérature («lu parce que cela donne des frissons et émeut l'imagination »), et les vraies news (« parce que cela comporte un message urgent qui invite à agir »). Dans la tradition américaine, le journaliste n'est pas celui qui agit mais qui donne des raisons et des moyens d'agir ; sur ce point encore le public journalism semble confondre les rôles du journaliste et du citoyen, ce qui, évidemment ouvre une autre voie, permise par l'internet, celle de faire du citoyen un journaliste (Gillmor, 2004).

\section{Du public journalism aux weblogs d'actualité}

Le public ou civic journalism fut d'abord un journalisme « local », tendant à resserrer les liens d'une communauté dont les questions semblaient négligées à la fois par les autorités et par les journalistes. II n’a jamais

27 Pour R. E. Park (1938), les « faits » n'existent par conséquent que dans le discours. 
vraiment atteint les grands journaux ni les grands réseaux de télévision. Mais il est le point de départ de deux phénomènes plus récents, et plus vastes, que sont les blogs ou les portails citoyens, et les accords de partenariat entre de grandes institutions d'informations et des portails civiques, à l'instar de l'Associated Press (AP) avec le portail canadien NowPublic.com en février 2007 (AP distribuera en syndication des nouvelles produites par le portail civique dans ses fils de presse), ou celui du partenariat entre Yahoo et Reuters (qui permet de mettre en ligne des photos et vidéos susceptibles d'être reprises sur les fils de Reuters). Plus encore, l'idée du « tous journalistes » a fait du chemin depuis les attentats contre le World Trade Center, la guerre contre l'Irak, le tsunami en Indonésie, les attentats de Londres, etc., et cela comme remède aux difficultés de l'information contemporaine, à un point tel que le très officiel rapport Tessier ${ }^{28}$ remis au ministère de la Culture envisage, parmi les remèdes à la crise de la presse française, une rémunération des citoyens informateurs sur le modèle ancien des correspondants de la presse locale et régionale. Donc, l'idée d'un journalisme citoyen a fait d'importants progrès, mais ce vocable unique recouvre trois réalités bien différentes.

II y a d'abord le fait que des citoyens ordinaires deviennent des «sources》 d'informations brutes pour les medias qui s'en emparent, comme le «téléphone rouge » d'Europe I. C'est le crowd sourcing de Jay Rosen. Mais les technologies de l'information et de la communication (TIC) ont amplifié le phénomène, en permettant à la source d'être son propre diffuseur de textes ou d'images. La nouveauté tient donc à la « dé-médiation » des organes de presse, et autorise aussi bien la diffusion d'informations tenues cachées par les autorités (les exactions à la prison d'Abou Ghraib), que les plus folles rumeurs. On doit constater une forte propension des « citoyens ordinaires » à prendre la parole, ce sur quoi repose une part du succès de la radio, et cela est un premier indice fort d'une « crise de la représentation ».

On note aussi la production d'informations par des citoyens plus «compétents » qui diffusent des contenus de qualité. C'est le cas d'Agoravox qui réussit là où Place Publique a échoué, cela sur le modèle du pionnier coréen Ohmynews. Ces organes comportent des instances de tri, qui ne sont pas exactement des rédactions en chef, puisqu'elles ne pilotent pas en amont la recherche d'information, mais les réorganisent en aval et les hiérarchisent plus ou moins sur leurs sites. Les current events blogs sont désormais une quasi institution (Le Cam, 2006). Les journalistes assument ici la seule fonction d'éditeurs d'une parole privée qu'ils rendent publique. $\|$ y enfin la diffusion d'information par des « experts » (dont une bonne part

${ }^{28}$ www.culture.gouv.fr/culture/actualites/rapports/tessier 
de journalistes), qui profitent de la baisse des coûts d'édition permise par l'internet. Le cas de rue89.com animé par d'anciens journalistes de Libération appartient évidemment à cette catégorie, comme la plupart des sites des grands organes de presse. Le très rapide succès de certains de ces sites (en matière de rentrées publicitaires) est encore trop récent pour assurer qu'un nouveau modèle économique se met en place. Mais il est certain que la précarité croissante du métier de journaliste a constitué un puissant accélérateur du mouvement. La responsabilité, ici, est celle du franc-tireur.

Dans tous les cas, l'ambition est bien de contribuer à un débat social en renouvelant les locuteurs légitimes, et en mettant en cause le monopole des « experts » ou des élus : le site d'Étienne Chouard à l'occasion du référendum français sur le traité européen en est un exemple tout à fait significatif. Mais un certain nombre de problèmes subsistent :

- le plus visible est l'instrumentalisation du « public », à des fins « journalistiques », sensible dès les débuts du public journalism. Ce «public » est toujours considéré comme une donnée, mais n'est jamais questionné, ni problématisé. La critique de Pierre Bourdieu (1973) ou Patrick Champagne (1990) sur l'opinion publique n'a donc rien perdu de son actualité. Plus encore, la figure du public devient une ressource pour légitimer la position d'acteurs émergents, y compris dans le monde des acteurs politiques (Ségolène Royal) ;

- du fait de la pseudo-communauté des internautes, les clivages sociaux sont fortement sous évalués. La critique marxienne n'a rien perdu de sa force ;

- la confusion entre journalisme et fonction représentative est extrêmement forte, et contribue paradoxalement à diminuer l'importance, dans les démocraties contemporaines, des « corps intermédiaires ».

La démocratie directe a toujours été un fantasme renouvelé par l'émergence de nouveaux médias, et on sait assez, depuis les travaux de Nicole Loraux (1997), que la démocratie athénienne était au service de groupes très majoritaires. Ce qu'on voit aujourd'hui est l'émergence de nouvelles revendications majoritaires ou communautaires, et que son moteur, fortement théorisé par Jürgen Habermas comme ré-féodalisation, est le ressort économique et marchand de la «marque » : la marque de tel organe d'information est une garantie, disent les détracteurs des sites émergents, mais eux même imposent leur marque, devenue le site de $\times$ ou de y, d'où le renforcement du rôle des propriétaires (Hoyer, Lauk, 2003).

\section{Conclusion}

Actuellement, la nature de la responsabilité des journalistes ne peut se penser qu'à partir de la perte de leurs repères traditionnels (Charron, 1993). Outre les changements globaux du monde déjà signalés et un relatif silence des intellectuels souvent dénoncé, ce sont les journalistes d'information générale et politique qui semblent les plus désorientés, 
du fait de leur perte d'influence sensible au profit de l'audiovisuel et de l'information spécialisée. Ce phénomène très général conduit à des réponses variables selon les lieux. La tradition américaine d'un journalisme tourné vers l'extérieur, le public, le citoyen, cherche d'autres façons de traiter les faits ou la mise en lumière de ce qui est caché ou masqué. La tradition française pose de façon plus nette la responsabilité du journaliste dans la vie de la cité, ne serait qu'en lui accordant une part active dans le débat d'idées ; il s'agit toujours de chercher où se situer par rapport au sens de l'histoire ou au progrès. Mais la légitimité est bel et bien en crise. Le journalisme audiovisuel dont l'ambition majeure est de transmettre le plus immédiatement possible les éléments d'une situation semble moins gêné parce qu'il se conçoit moins comme médiateur que comme passeur, et le journaliste d'information spécialisée peut se réfugier dans sa technicité.

Au fond, c'est à une redéfinition de l'identité journalistique que ce sujet invite: «Depuis la fin des années 80, le phénomène du journalisme public semble fondé sur l'idée que les journalistes ont pour mission de permettre aux citoyens de débattre des questions publiques qu'ils estiment fondées [...]. Le développement des current events blogs semble prolonger ces pratiques, mais, et c'est ce qui est novateur, en les transférant du groupe des journalistes traditionnels vers le public lui-même » (Le Cam, 2006 : 152). C'est pour cela que l'actuelle « crise du journalisme » semble bien provenir d'une crise plus générale de la représentation.

\section{Références}

Abare McCane A., 1992, «L'indépendance et le journaliste », Médiaspouvoirs, 26, pp. 5- 14.

Althusser L., 1970, « Idéologie et appareils idéologiques d'État », La pensée, I5 I, repris in : Positions, 1976, et dans Sur la reproduction, Paris, Presses universitaires de France, 1995, ch.VI, «L'État et ses appareils 》, pp. I 01 - I 24

Arendt H., 1978, Juger. Sur la philosophie politique de Kant, trad. de l'anglais par M. Revault d'Allonnes, Paris, Éd. Le Seuil, 1991.

Atton C., 2002, Alternative media, London, Sage.

Bagdikian Ben H., 1983, The Media Monopoly, Boston, Beacon Press.

Barnouw E. et al., 1997, Médias et conglomérats, New York, The New Press, trad. de I'américain par C. Deniard, Paris, Liris, 2005.

Bly N., I 887, Ten Days In A Mad House, New York, Norman L. Munro Publisher.

Bourdieu P., 1973, «L'opinion publique n'existe pas », Les temps modernes, 318 , pp. 1292- 1309 (texte d'une conférence faite au cercle Noroit en janvier 197| et reproduite dans Questions de sociologie, Paris, Éd. de Minuit, 1980, pp. 222-235). 
Brin C., Charron J., de Bonville J., 2004, Nature et transformation du journalisme, Québec, Presses de l'université Laval.

Champagne P., 1990, Faire l'opinion, Paris, Éd de Minuit.

Charity A., 1995, Doing public journalism, New York, Guilford Press Inc.

Charon J.-M., 1993, Cartes de presse, Paris, Stock.

Chartier R., 1990, Les origines culturelles de la Révolution française, Paris, Éd. Le Seuil.

Fishman M., 1988, Manufacturing the News, University of Texas Press.

Gillmor D., 2004, We the media, Grassroots Journalism by the People for the People, O'Reilly Inc.

Habermas J., 1962, L'espace public. Archéologie de la publicité comme dimension constitutive de la société bourgeoise, trad. de l'allemand par Marc B. de Launay, Paris, Payot, 1978.

Hoyer S., Lauk E., 2003, «The Paradoxes of the Journalistic Profession », Nordicom Review, 24 (2), pp. 3-18.

Kant E., 1784, 1786, Qu'est-ce que les Lumières?, Que signifie s'orienter dans sa pensée ?, trad. de l'allemand par J. Darbellay, Paris, Flammarion, 1996.

Lambeth E. B., 1998, « Public journalism as a Democratic Practice », pp. 15-35, in : Lambeth et al., eds, Assessing public journalism, University of Missouri Press.

Le Cam F., 2006, «États-Unis: les weblogs d'actualité ravivent la question de l'identité journalistique », Réseaux, | 38, pp. I39-I58.

Loraux N., 1997, La cité divisée. L'oubli dans la mémoire d'Athènes, Paris, Payot.

Meritt D., 1995, Public Journalism and Public Life: Why Telling the News is not enough, New York, Hillsdale, Lawrence Erlbaum Associates.

Moloch H., Lester M., 1974, «Informer: une conduite délibérée de l'usage stratégique des événements », American Sociological Review, 39, trad. de I'américain par M. Chr., Gamberini, in : Réseaux, 75, janv.-févr. 1996, pp. 23-4I.

Muhlmann G., 2004, Du journalisme en démocratie, Paris, Payot.

Park R. E., 1938, « Reflexions on Communication and Culture », American Journal of Sociology, XLIV, pp. I87-205.

- 1942, The crowd and the Public, Chicago, University of Chicago Press, 1972 (trad. américaine par Ch. Elsner du texte original Masse und Publicum, écrit en 1904).

Prodhomme M., 2003, La place du discours sur l'éthique dans la construction de l'espace et de l'identité professionnelle des journalistes, thèse en sciences de l'information et de la communication, Université Lyon 2.

Reynié D., 1998, Le triomphe de l'opinion publique. L'espace public français du XV| au $X X^{e}$ siècle, Paris, O. Jacob.

Rieffel R., 1984, L'élite des journalistes, Paris, Presses universitaires de France.

Rosen J., 1999, What are journalists for?, New Haven, Yale University Press. 
Siebert F. S., Peterson T., Schramm W., 1963, Four theories of the press. The authoritarian, libertarian, social responsability and Soviet communist concepts of what the press should be and do, Urbana, University of Illinois Press.

Simmel G., 1908, Soziology, chap. 4, trad. de l'allemand par S. Muller sous le titre Le conflit, Belval, Circé, 1992, 1995.

Tarde G., 190 I, L'opinion et la foule, Paris, Presses universitaires de France, 1989.

Tétu J.-Fr., 2002, « Éthique journalistique et identité professionnelle. Le syndicat national des journalistes de 1918 à 1936 », pp. 195-204, in: Dragàn I., dir., Nouvelles technologies et redéfinition des territoires de la communication, Bucarest, Ed.Tritonic.

Tuchman G., 1978, Making News. A study in the Construction of Reality, New York, Free Press.

Véron É., 1987, La sémiosis sociale, Paris, Presses universitaires de Vincennes SaintDenis.

Watine T., 2003, « Le modèle du "journalisme public" », Réseaux, 35, pp. 23 I-239.

Weaver D. H., Wilhoit G. C., 1986, The American journalist: a Portrait of U.S. news people and their work, Bloomington, Indiana University Press. 